\title{
Erratum - Daphnia magna fitness during low food supply under different water temperature and brownification scenarios
}

\author{
Andrea GALL, ${ }^{1,2 \#}$ Martin J. KAINZ, ${ }^{2}$ Serena RASCONI ${ }^{2 *}$ \\ ${ }^{1}$ Department of Limnology and Oceanography, University of Vienna; ${ }^{2}$ WasserCluster Lunz, Lunz am See, Austria \\ ${ }^{*}$ Current address: Institute for Chemistry \& Biology of the Marine Environment (ICBM) University Oldenburg, 26382 Wilhelmshaven, \\ Germany \\ *Corresponding author: serena.rasconi@wcl.ac.at
}

Received: August 2018. Accepted: August 2018.

\begin{abstract}
This corrects the article entitled "Daphnia magna fitness during low food supply under different water temperature and brownification scenarios" by the authors Andrea Gall, Martin J. Kainz and Serena Rasconi, published with DOI 10.4081/jlimnol.2016.1450. The data on somatic growth rates reported in the results section, paragraph "Life history traits", page 165, were incorrect and the rectified data are presented. Fig. 4 has also been corrected accordingly.
\end{abstract}

Daphnia somatic growth rate $\left(\mathrm{d}^{-1}\right)$ was lowest in $C$ $\left(0.009 \pm 0.003 \mathrm{~d}^{-1}\right)$, higher in $b\left(0.013 \pm 0.005 \mathrm{~d}^{-1}\right)$, again higher in $T\left(0.026 \pm 0.005 \mathrm{~d}^{-1}\right)$, and highest in $T b$ $\left(0.032 \pm 0.001 \mathrm{~d}^{-1}\right.$, Fig.4), and significantly higher in $T$ and $T b$ from $C$ and $b$ (ANOVA with Tukey's HSD test; $\mathrm{P}<0.01$ ). The final biomass accrual of Daphnia was $4.59 \pm 1.86 \mu \mathrm{g}$ in $C, 7.52 \pm 3.36 \mu \mathrm{g}$ in $b, 17.3 \pm 5.29 \mu \mathrm{g}$ in $T$, and $21.2 \pm 2.31 \mu \mathrm{g}$ in $T b$, and significantly higher in $T$ and $T b$ from $C$ and $b$ (ANOVA with Tukey's HSD test; $\mathrm{P}<0.01$ ).

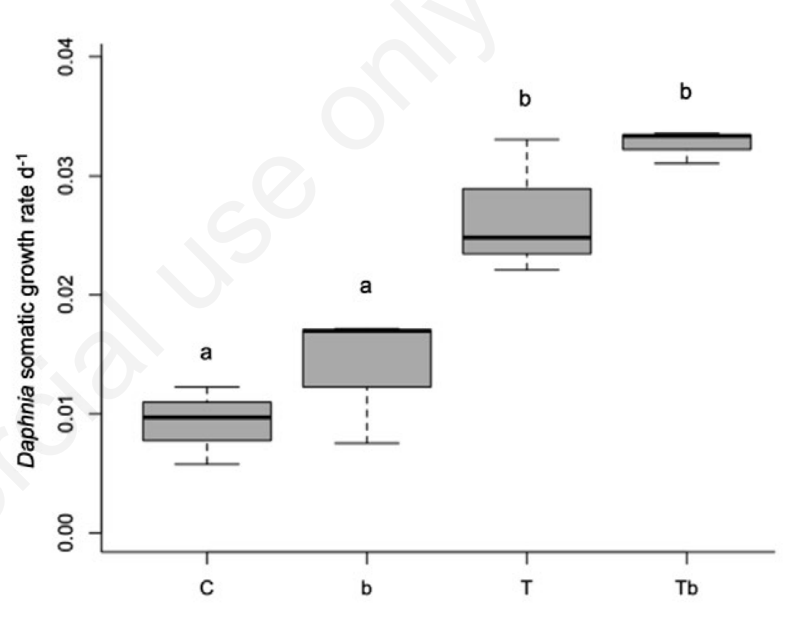

Fig. 4. Daphnia somatic growth rate $\left(\mathrm{d}^{-1}\right)$ during the experiment in the four treatments: control $(C)$, brownification $(b)$, temperature $(T)$, and temperature $\mathrm{X}$ brownification $(T b)$. The box borders indicate the lower and upper quartiles, the centerline is the median and the whiskers extending out from indicate the most extreme data point outside the upper and lower quartiles. Different letters account for statistical significant difference (ANOVA with Tukey's HSD test $\mathrm{P}<0.05$ ). 